\title{
The Study of Psychopathology from the Network Analysis Perspective: A Systematic Review
}

\author{
Alba Contreras ${ }^{a}$ Ines Nieto ${ }^{a}$ Carmen Valiente $^{a}$ Regina Espinosa ${ }^{b}$ \\ Carmelo Vazquez ${ }^{a}$ \\ aDepartment of Clinical Psychology, School of Psychology, Complutense University, Madrid, Spain; \\ ${ }^{b}$ Department of Psychology, School of Education and Health, Camilo José Cela University, Madrid, Spain
}

\section{Keywords}

Network analysis · Network theory · Systematic review ·

Psychopathology $\cdot$ Mental disorder

\begin{abstract}
Background: Network analysis (NA) is an analytical tool that allows one to explore the map of connections and eventual dynamic influences among symptoms and other elements of mental disorders. In recent years, the use of NA in psychopathology has rapidly grown, which calls for a systematic and critical analysis of its clinical utility. Methods: Following PRISMA guidelines, a systematic review of published empirical studies applying NA in psychopathology, between 2010 and 2017, was conducted. We included the literature published in PubMed and PsycINFO using as keywords any combination of "network analysis" with the terms "anxiety," "affective disorders," "depression," "schizophrenia," "psychosis," "personality disorders," "substance abuse" and "psychopathology." Results: The review showed that NA has been applied in a plethora of mental disorders in adults (i.e., 13 studies on anxiety disorders; 19 on mood disorders; 7 on psychosis; 1 on substance abuse; 1 on borderline personality disorder; 18 on the association of symptoms between disorders), and 6 on childhood and adolescence.
\end{abstract}

Conclusions: A critical examination of the results of each study suggests that NA helps to identify, in an innovative way, important aspects of psychopathology like the centrality of the symptoms in a given disorder as well as the mutual dynamics among symptoms. Yet, despite these promising results, the clinical utility of NA is still uncertain as there are important limitations on the analytic procedures (e.g., reliability of indices), the type of data included (e.g., typically restricted to secondary analysis of already published data), and ultimately, the psychometric and clinical validity of the results.

(c) 2019 S. Karger AG, Basel

\section{Introduction}

The epistemological bases of classification and conceptualization of psychopathology are being increasingly subjected to scrutiny $[1,2]$. Some authors, from very different perspectives, cogently argue that [3-5] making advances on the science of psychopathology and its treatments can be seriously hindered if these insufficient diagnostic systems are used $[6,7]$. In recent years, the network approach has been gaining popularity in the field of psychopathology as one of the alternatives to the

\section{KARGER}

(c) 2019 S. Karger AG, Basel

E-Mail karger@karger.com

www.karger.com/pps
Carmen Valiente

Faculty of Psychology, Complutense University

Pozuelo de Alarcón

ES-28223 Madrid (Spain) 
classification systems [8]. Categorical approaches like DSM and some alternatives such as the Research Domain Criteria system assume an underlying biological condition as the main cause of mental disorders in which all symptoms within a category have the same diagnostic weight. This idea has been criticized $[9,10]$. In contrast, network models recognize that symptoms can cause other symptoms, resulting in consistent profiles or syndromes $[11,12]$. From the network perspective, mental disorders are seen as conditions consisting of strongly connected symptom networks with no assumption of a latent entity subsumed under the symptoms [13]. Interestingly, this emphasis focuses on specific symptoms, or patterns of symptoms, and is conceptually connected to the Clinimetrics Approach initiated more than 3 decades ago $[5,14]$. A common feature of Network Analysis (NA) and Clinimetrics is that both approaches are critical of the traditional use of sum-scores in scales to assess psychopathology as this practice includes redundant or noninformative items and, ultimately, may have scarce validity and clinical utility $[5,15]$. Thus, NA may contribute with the aim of finding informative or key symptoms associated to the clinical status or the prognosis of patients, rather than relying on global scores from scales [16] or categorical diagnosis.

One of the advantages of NA is that the interconnections of symptoms can be mathematically analyzed and visually represented. From a topological point of view, a network structure consists of nodes that represent the variables studied and edges orlines that connect nodes and represent the relationship between them [12]. Graph theory has been used to represent different spatial and functional characteristics that reveal information about the type of relationship between the nodes in the network.

One of the most novel features of NA is that it provides several centrality measures that allow to identify symptoms with the greatest importance in the network structure This approach may shed light on the dissatisfaction of clinicians and researchers with standard psychometric approaches, as it could help identify specific symptoms that convey the highest level of clinical information [5, 15]. Furthermore, phenomena such as comorbidity are explained as the interconnection, through "bridge" symptoms, between different groups or subgroups of symptoms, without having to allude to covariation between different latent entities [8]. Thus, network perspective and NA may provide some useful conceptual and analytical tools to describe psychopathology and to explore issues related to the structure of psychological problems as well as their onset and maintenance.
NA has been used to explore complex systems across different fields [17]. The application of these novel tools in psychopathology is relatively new, and it has already received considerable attention and recognition $[8,18]$. Its conceptual beginning took place in $2008[18,19]$, while its empirical foundation dates back to $2010[18,20]$. In recent years, solid efforts have been made to develop statistical models that allow the estimation of psychopathological networks and procedures to assess the accuracy of network parameters and measures [12, 21-23]. Network theory and NA are rapidly growing, not only regarding methodological issues, but also in providing intuitively appealing explanations of psychopathological phenomena $[18,22]$.

The aim of this paper is to provide a systematic review of empirical research (i.e., use of clinical data) that has used NA to study psychopathology. Given the growing number of studies in the field, it seems appropriate to provide a general overview of the strengths and limitations of this approach in the clinical psychology field. The present review aims to summarize the network studies on psychopathology in terms of their main characteristics (i.e., sample type and characteristics, instruments used to assess psychological variables or nodes, type of network estimated, robustness of the analysis), and given the current debate of replicability in psychology [24], sharing of software codes and/or databases were also coded.

\section{Methods}

Search Strategy

Following the PRISMA guidelines [25], a systematic literature search was carried out using PubMed and PsycINFO databases. Specific keywords are fully described in Figure 1. Eating disorders were excluded since there has been a recent overview of NA studies in this field [26]. The search was restricted to peer-reviewed studies published in English.

\section{Study Selection Process}

The selection process was carried out by two of the authors (A.C. and I.N.), applying the following eligibility criteria: (a) empirical studies; (b) paper version published from January 2010 to December 2017); and (c) inclusion of measures of psychopathological symptoms (in general or clinical population). Network studies that did not focus on clinical symptoms were excluded (e.g., well-being). In addition, the bibliography of relevant papers was manually revised to complete the search (Fig. 1).

\section{Data Extraction}

For the included studies, the following information was collected: (a) sample characteristics; (b) network elements and instruments used to assess psychopathology; (c) type of data (cross-sectional or longitudinal data); (d) NA information provided (type of
Contreras/Nieto/Valiente/Espinosa/ Vazquez 


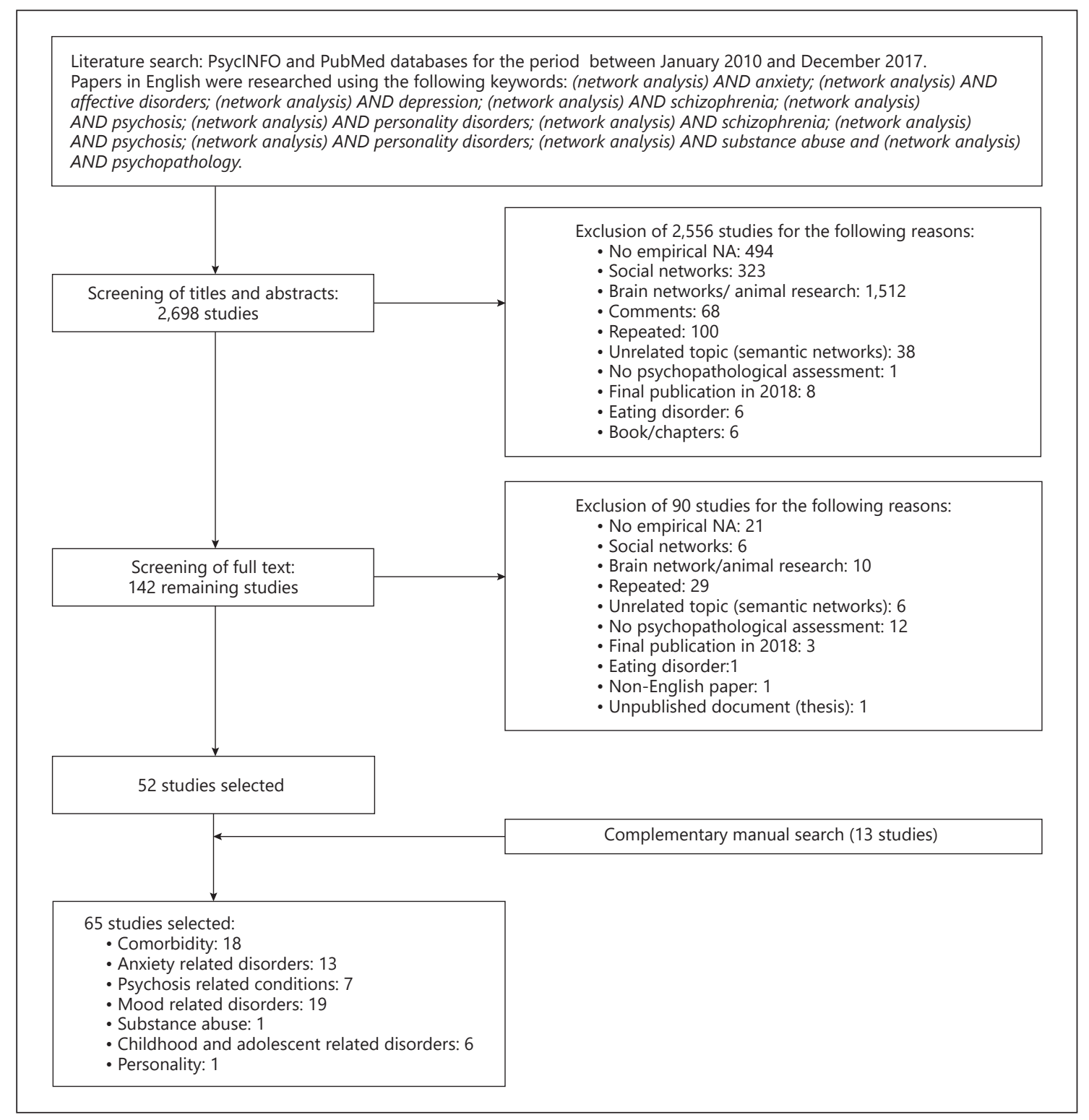

Fig. 1. Process of literature search strategy of network analysis (NA) studies in psychopathology.

network estimated, centrality metrics, and robustness analyses) availability of software code or syntax (e.g., R script) and/or data (i.e., correlation matrix or datasets) in the published paper (online suppl. Tables 1-3; for all online suppl. material, see www.karger. com/doi/10.1159/000497425).

\section{Results}

\section{Study Selection and Characteristics}

A total of 52 studies met the criteria and were selected for the current revision. Moreover, 13 relevant articles were added from manual complementary cross-referencing search. As a result, a total of 65 studies were included (online suppl. Tables 1-3). The majority of the literature reviewed has been published recently (23 studies in 2016, 28 studies in 2017). Overall, most of the studies used adult samples; only 8 studied NA in a childhood and/or adolescent population. In terms of the type of data, 46 studies used cross-sectional data, while only 19 studies used longitudinal data (7 of them using Experience Sampling Methodology [ESM]). Regarding the type of NA, 23 studies constructed a Directed Network while the remaining used association networks or Regularized Partial Correla- 
tion Networks, and 55 studies calculated centrality indexes. (See further details in online suppl. Materials). Only 21 studies assessed robustness or quality of estimated parameters (stability and/or accuracy of results) but tools to carry out these procedures have been available recently [27]. Finally, a low number of studies shared their data in the published paper (i.e., 18 shared correlation matrixes and only 6 shared both data and scripts).

\section{Results of NA for Specific Mental Disorders}

Post-Traumatic Stress Disorder

The literature identified 10 NA studies on post-traumatic stress disorder (PTSD; online suppl. Table 1). Across studies, results disclosed strong associations among avoidance symptoms $[28,29]$ and between hypervigilance and startle response [28, 30-33]. Particularly, McNally et al. [34] found that physiological responses to reminders of trauma predicted symptoms such as "being upset" "flashbacks," and "traumatic dreams," in a sample of adults who reported histories of childhood sexual abuse. According to these authors, physiological reactivity, which is associated in NA with being female [32], may play a potential causal role in the activation of the PTSD network. Moreover, NA has also found some unexpected interconnections between anger, sleep, and concentration problems, suggesting that difficulties in the regulation of emotions and attention may arise from sleep-related problems in trauma-related problems [28]. Likewise, Glück et al. [35] showed that "trait anger," "rumination anger," and "emotional abuse" may play an important role in persons who suffered childhood trauma, highlighting the possible clinical implications of including anger in trauma interventions.

Interestingly, 1 study showed that connections may vary in different age groups, that is, symptoms such as "amnesia" and "numbness of negative effect" were more strongly associated in children than in the adolescents [36]. In addition, in the only longitudinal study that compared networks across time, Bryant et al. [31] found that, after 1 year, re-experiencing symptoms were more strongly connected than during the acute phase and that physiological reactivity was strongly associated with startle response which, in turn, was associated with hypervigilance. This strong "reactivity association," according to these authors, could be conceptualized as a circuit of fear that becomes increasingly more sensitive to long-term threats.

NA has also shown PTSD symptom connections with other relevant elements and clinical problems, such as depression symptoms [30] or "low social support after the exposure," which has been found to be connected with sleep disturbances in individuals with PTSD [32]. Finally, NA has revealed mixed results regarding central symptoms in PTSD. For instance, "physiological reactivity" and "flashbacks" have been found to be central symptoms in veteran and earthquake survivors $[28,30]$. However, central symptoms in terrorist attack witnesses are "emotional numbness" and "concentration difficulties" [32], whereas intrusive thoughts and anger were highly central in a sample of witnesses from a shooting event [29]. Again, after a year from a traumatic injury, "re-experiencing" persisted as a core symptom [31], whereas "emotional cue reactivity" is the most important node in a sample of refugees [33]. Lastly, Jayawickreme et al. [37] found that war social problems were more important than the traumatic event in a sample of war survivors.

In sum, PTSD results provide relevant information about elements interactions, about variables that may affect symptom connections and have allowed the finding of some unexpected associations among symptoms [28] that deserve to be further explored. Findings also illustrate potential pathways between traumatic events and symptoms [37]. However, the heterogeneity of the results may be due to differences in the type of traumatic experiences or in the size and type of samples included in the studies, making it difficult to integrate the results and imposing caution on the generalizability of the results.

\section{Anxiety-Related Disorders}

Two studies focused on social anxiety (SA) symptoms interaction and other non-symptoms variables (online suppl. Table 1). Tsuruta et al. [38] innovatively examined the association among SA symptoms and cognitive functioning and found that orienting attention to non-emotional material was linked to fear of social situations, which impacted the social experience by triggering avoidance of social behaviors. NA has also been used to study less known anxiety syndromes such as olfactory reference syndrome. Tsuruta et al. [38] study revealed that SA may play a key role in the onset of fear to bodily odors.

Finally, 1 study explored anxiety related to death in patients suffering from cancer [39], disclosing that the most central concern related to death is "running out of time." The authors also identified nodes that act as a bridge between 2 death-related anxiety clusters (i.e., 1 related to practical fears regarding the process of dying, and the other to existential concerns). These findings suggest that psychology interventions should be aimed at targeting those central symptoms to alleviate anxiety related to death.
Contreras/Nieto/Valiente/Espinosa/ Vazquez 
Mood-Related Disorders

The literature search revealed a total of 19 studies on mood symptom networks: 16 on depression, 1 on suicide attempters, 1 on bipolar disorder, and 1 on alexithymia (online suppl. Table 1).

Bringmann et al. [40] found that positive emotions were negatively associated with negative mood variables, that the presence of one symptom predicted the occurrence of the same symptom in the future and that depressed females showed stronger associations than controls. Also, using ESM methods, Pe et al. [41] have shown that, compared to healthy controls, participants with major depression had higher network density of negative emotions but there were no differences in regard to the density of positive emotions. These results suggest that in depression previous negative emotions have a greater influence on the next negative emotional states, which make negative emotions more resistant to change in this disorder [41].

NA has also been used to study the interaction of depressive symptoms over time [93]. It was found that patients with persistent depression showed stronger associations between depressive symptoms than those in remission, supporting the idea that the strength of connections is associated to vulnerability [42]. Likewise, Madhoo and Levine [43] reported that the connectivity among symptoms significantly diminished after an intervention. Several studies have used ESM to collect timeseries data in depressed participants, although, the outcomes assessed are heterogeneous. For example, Dejonckheere et al. [44] found that the perception of social pressure to feel good instigated increases in slowed-down symptoms of depression (e.g., hypersomnia, motor retardation). More interesting, from a comprehensive view of psychopathology, is that several studies added non-symptom variables in the network (online suppl. Table 1). Hoorelbeke et al. [45] studied the potential role of risk and protective factors and found that resilience was the principal hub in the network and it could be a key factor in the remission of depression. Cramer et al. [46] found specific connections between depressive symptoms (e.g., "feelings of worthlessness") and stressful life events such as "ending of a romantic relationship." In a sample of women in their third trimester of pregnancy, Santos et al. [47] assessed the relationship between stress and reproductive biomarkers (e.g., cortisol) and depression. Interestingly, all biomarkers showed very small associations with symptoms of depression which, according to the authors, may cast some doubts about their role as causal candidates of symptoms of depression.

NA and Psychopathology
Three studies have also explored depression and personal losses. Robinaugh et al. [48] and Fried et al. [49] found associations between loss of a spouse and depression symptoms. Specifically, "loneliness" was found to activate other symptoms through its association with loss [49] and to connect to risk factors, such as lack of instrumental social support [48]. Maccallum et al. [50] analyzed the networks of depressive and complicated grief symptoms regarding 2 different types of losses (i.e., death of a spouse or a parent) in general population. The results showed that both types of losses produced very similar networks in which the strongest connection is the link between yearning and emotional pain. Remarkably, pain avoidance had a peripheral situation in both networks.

Regarding centrality, Fried et al. [51] found that DSM5 criteria symptoms (e.g., sad mood) were not more central than non-DSM symptoms (e.g., anxiety). In clinical, as well as general populations, some authors have found that "concentration problems" and "feeling sad" were central symptoms $[42,52]$. These results are in line with the findings of the study by McWilliams et al. [53] in participants suffering from chronic pain, showing that difficulty in concentrating, loss of interest, depressed mood, and fatigue were the most important symptoms.

De Beurs et al. [54] analyzed suicide ideation in a sample of patients following a suicide attempt, revealing that "desire for an active attempt" was the most central symptom of the network for the entire sample. One study focused on bipolar disorder [55], finding that "loss of energy" was highly central in bipolar patients with different levels of severity and patterns of symptoms. Yet, there were specific variations, in terms of centrality, in different subtypes of patients. The highest degree of centrality was "increased speech" and "loss of interest" in the minimally impaired group, "decreased self-esteem," and "slowness" in the depressed group and "restlessness" and "suicidality" in the cycling group [55]. Watters et al. [56] investigated the basic components of alexithymia and their mutual interaction. They found that the strongest associations were between difficulties in identifying and describing feelings, and that both components were also the most central ones in alexithymia.

Some preliminary findings on the connectedness of symptoms in depression are worth mentioning. For instance, the finding that depressed individuals show a strong connection of symptoms of depression [47] or emotions [41] could be relevant to explore the organization of symptoms in depression. 
Psychosis-Related Conditions

The literature search revealed a total of 7 studies that focused on psychosis-related symptoms (online suppl. Table 1). A "transdiagnostic network approach" was used to study psychosis and to analyze multiple domains of psychopathology $[57,58]$. Associations were found within each psychotic domain $[57,58]$, which were especially strong for negative symptoms [57] and within different domains For example, there are interconnections between some positive symptoms (e.g., "being persecuted") and anxiety items (e.g., "worried about panic"), negative symptoms (e.g., "lack of energy"), and depression items (e.g., "feeling tense") [57]. Their NA topology discloses the possibility that, once the delusion is activated, it triggers anhedonia symptoms, which in turn activates depressive symptoms or vice versa.

Using ESM data in a single participant through different disorder course moments, it was found that when the patient has not relapsed yet, "feeling down" and "paranoia" fuelled each other (i.e., the lower the mood, the higher the paranoia in the next moment) [59]. However, this association was weaker in the full relapse group, while paranoia was directly connected to hearing voices. NA can also be used to explore changes in patters of symptoms before and after interventions. Levine and Leucht [60] studies negative symptoms in 3 different moments (i.e., before, after, and during an intervention). They identified a "negative symptoms severity system" in which symptoms severity are grouped in 4 clusters (e.g., "affect," "poor responsiveness," "lack of interest," and "apathy-inattentiveness"). They also highlighted 2 central symptoms ("decreases spontaneous movement" and "speech") as potential future treatment targets, due to the fact that they remained central after and during intervention, respectively. Similarly, Esfahlani et al. [61] compared the symptoms network at baseline and at 18 months' follow-up of a trial of antipsychotics in individuals with psychosis. Results showed that the treatment responsive group had more densely connected symptoms after the treatment, while global connectivity of the treatment resistant group is not affected by the treatment [61].

Two of the studies reviewed in this section added adversity variables in the networks $[62,63]$. In a sample of general population, developmental trauma was found to be connected to psychotic expression and somatization [62]. Further, the authors found that drug use might play a mediating role between trauma experienced and the onset of psychotic symptoms [62]. Moreover, in a sample of people diagnosed with a psychotic disorder, childhood trauma was associated with positive and negative symp- toms only through some general psychopathology symptoms, such as anxiety, suggesting that there may be different pathways between trauma and psychosis [63].

Both cross-sectional $[57,58,60]$ and longitudinal studies $[59,60]$ seem to be pointing to the significant role that negative effect plays in paranoia as well as the possibility to map transdiagnostic symptoms of psychopathology [57].

\section{Personality Disorders}

A study on borderline personality disorder (BPD) was identified in the present literature review (online suppl. Table 1). Authors compared the relation of 9 characteristics of BPD in 2 different samples (university students and clinical population) [64]. Although "affective instability," "identity disturbances," and "effort to avoid abandonment" appeared to have a central role in both samples, some edges were unique for the clinical sample (i.e., suicidal behavior and unstable relationship) that highlights particular connections between symptoms in severe manifestations of BPD.

\section{Substance Abuse Disorders}

The literature search revealed only 1 NA study in a sample of people who reported "having used substances more than 6 times in their lifetime" (online suppl. Table 1). Rhemtulla et al. [65] found that "using a substance more than planned" was strongly connected to "tolerance." Besides, a common association between "being unable to stop" and "hazardous use" was found when comparing symptoms connections across different substances. However, some correlations changed across substance networks. For example, hazardous use and legal consequences were strongly connected when using sedatives, but it was not for the opioids, cocaine or hallucinogens networks. Regarding centrality, "substance used more than planned" was the most central symptom in the general substance use network, as well as in the cocaine, cannabis, and stimulants networks, which may indicate that losing control over a drug may precipitate a host of other types of abuse and dependence symptoms.

\section{Results from NA in Psychopathology and/or Comorbidity}

The literature search revealed a total of 18 studies on general psychopathology and/or comorbidity (online suppl. Table 2).

\section{Comorbidity with Depression}

Out of the 9 studies that focused on comorbidity with depressive symptoms, some have analyzed comorbid
Contreras/Nieto/Valiente/Espinosa/ Vazquez 
MDD and GAD [66-69]. All of them found a highly connected network in which no symptom remained isolated from the others. Regarding centrality, studies have reached different conclusions. While 1 study found that "worry" and "sad mood," the main diagnostic criteria for GAD and MDD, respectively, were the most central in a cross-sectional network [68], another study found that these symptoms were the least central in a temporal network [69]. However, both studies pointed out "anhedonia" and "guilt" as highly central in their comorbid networks.

Two studies analyzed depression and PTSD [70, 71] and found that symptoms of depression and PTSD formed separated clusters but they were also connected by bridge symptoms, such as "sleep disruption" or "concentration difficulties" (online suppl. Table 2).

Regarding comorbid depression and psychosis, some authors found that symptoms formed 2 separated clusters, with the symptom of paranoia more closely related to depression [72]. Using longitudinal data, Wigman et al. [73] studied the dynamics of 5 mental states (i.e., cheerful, insecure, content, down, and suspicious) in individuals with a diagnosis of depression, psychosis, and no diagnosis. The main difference between the clinical groups was that there were many connections between positive and negative emotions in the depression group, while they formed 2 separate clusters in the psychosis group. The highest centrality indexes were down momentary mental states for the psychosis group and positive momentary mental states for the healthy controls.

Finally, studying comorbid depression and obsessivecompulsive disorder (OCD), McNally et al. [74] found that both clusters of symptoms were connected through "sadness," but sleep and appetite symptoms were not connected to either depression or OCD. These types of results illustrate that not all symptoms play the same role when developing comorbid disorders.

\section{Comorbidity between Different Conditions}

The reviewed literature identified 9 NA studies that focused on comorbidity and psychopathology (online suppl. Table 2). Two of them used temporal networks to explore the dynamics of emotions with longitudinal data. It was found that people with high neuroticism and healthy people had denser emotion networks in comparison with people low in neuroticism or depressed people, respectively. Authors tried to see the relationship between neuroticism and the centrality in the networks and their variability, but they arrived at different conclusions $[75,76]$, remaining unclear how this trait influences emotional changes in time.

NA and Psychopathology
Some studies have used NA to explore the association between symptoms classified within different diagnostic categories [77-80]. The main findings have consistently been that some symptoms cluster together (e.g., symptoms of depression) [78], but are also connected by bridging symptoms. For example, "internal avoidance" and "identity disturbance" are related in comorbid PTSD and BPD [79], while "drinking to cope" and "subjective stress" could explain comorbid Alcohol Use Disorder and anxiety and depression [80]. These results may be important in terms of identifying individuals at risk to develop two or more disorders and also to be used in treatment to reduce current and future comorbidities.

Finally, NA has allowed exploring the relationship between psychopathological symptoms and non-symptom variables. Using longitudinal data, it was found that environmental factors (i.e., childhood trauma, urbanicity, cannabis use, and discrimination) increased symptom connectivity [81] and that "self-criticism" could play a central role in the relationship between rumination and executive control [82]. Further, personality variables such as extroversion and openness to experience were left outside the cluster of anxiety and depression, but extrovert personality together with symptoms such as "worry," "perceived distress" or "low energy" were found to be the most central in the network [83].

In sum, comorbidity is an interesting field to be studied using NA to discover how symptoms from different disorders are related. These findings have direct implications on their use in clinical interventions, as will be further explained in the discussion section.

\section{Results from NA in Childhood and Adolescents Related Disorders}

The literature search revealed a total of 6 NA studies that focused on disorders related to childhood or adolescence (online suppl. Table 3 ) in both of which, samples and outcomes studied were heterogeneous. Anderson et al. [84] studied autistic traits in a sample of children with Pervasive Developmental Disorder. They found that "usual eye contact" and "facial expression directed towards others" were the most important nodes. Moreover, they showed that anxiety was more central in males, and that social nodes were more central in low functioning children. Ruzzano et al. [85] studied the comorbidity between autism and OCD symptoms, revealing that compulsion symptoms may be the bridge between autism and OCD [85].

Two studies focused on attention deficit hyperactivity disorder (ADHD) symptoms interactions. Martel et 
al. [86] found that ADHD symptoms changed across time, although symptoms such as "often easily distracted" and "difficulty sustaining attention" remained central over time. Likewise, Smith et al. [87] showed an association between ADHD and oppositional defiant disorder symptoms and also that anger was the most important symptom in preschoolers with oppositional defiant disorder.

Boschloo et al. [88] examined the empirical network of 95 emotional and behavioral problems in a sample of adolescents. Findings revealed strong connections within psychopathological domains (i.e., externalizing, internalizing, attention, thought, and social problems), but also connections between domains. Finally, Hasmi et al. [89] studied whether the dynamics of emotions in daily life differed across different levels of genetic liability and exposure to childhood trauma in a general population mixed-gender twin sample. NA confirmed that negative emotions are associated with genetic risk factors.

\section{Discussion}

In the current context of crisis of traditional classification systems within mental health, NA research has substantially increased in recent years. The present systematic review revealed evidence that highlights NA as a promising psychopathological research tool for studying symptom connections. While it may be clinically useful and ultimately sensitive to changes in the clinical population, a number of important caveats also emerge, which will be addressed below.

NA has been used to study many psychological disorders (online suppl. Table 1-3). Our systematic review reveals that a great part of NA research has been conducted in an exploratory way of which mood disorders, PTSD, psychosis-related conditions, and comorbidity phenomena are the most studied areas. Comparing results of NA within a given domain of psychopathology is problematic as the critical mass of studies using these tools is still scarce and studies are very heterogeneous in regard to sample characteristics, types of measures or other relevant variables. Thus, part of the problem with current research in NA is that it is too descriptive and results are rather diverse. Given the exponential growth of the literature in this field, and the heterogeneity of the results, it is expected that there will be specific systematic reviews for each psychopathology domain. Therefore, a systematic review may provide a good main entrance to this field of studies. Although NA in certain cases provides expected results, like the fact that sadness is an almost ubiquitous central symptom in depression $[42,43,52]$, the results of the present review supports the idea that NA adds information that the traditional classification models do not incorporate [88]. For instance, our review shows the potential capacity of NA in identifying unexpected associations between symptoms [28]. In the case of depression, NA has shown that a low level of energy often emerges as a core symptom in moodrelated disorders, which may predict the onset of major depression [52], albeit it is important to consider that the centrality of symptoms may vary at different time points through the course of the problem [43]. Though "fatigue" or "loss of energy" are not DSM symptoms, they emerged as central symptoms in depression [52] and bipolar patients [55]. In the case of PTSD, NA studies have identified a potential causal interconnection of anger with sleep and concentration problems, pointing the possibility that these associations may affect the regulation of emotions and attention [28]. Although most of the extant NA research has focused on symptom-tosymptom interactions, there is evidence showing that other non-symptom should be meaningfully incorporated in psychopathology networks. Our results bear out that adding non-symptom element can enhance the understanding of important aspects of psychopathology. The range of these elements included in NA vary a great deal, from attention bias in SA networks [90] to biomarkers [47], resilience in depressive networks [45] or the so-called external field variables [22], like life events [46]. For instance, the study by Isvoranu et al. [63] revealed multiple potential pathways between childhood trauma and psychosis, either through a pathway of emotional distress or through general psychopathology. As the field of NA matures, it is desirable that it reflects the complex interactions between different components that, beyond symptoms, are involved in the etiology and maintenance of psychopathology [8]. This implies that it is not possible to attribute a casual or explanatory priority to the psychology or biology, but rather that a holistic research strategy is needed [13], in which all the evidence-based elements involved in psychopathology up to now are taken into account.

NA may also cast some light on some of the current conceptual and classification dilemmas in psychopathology. For instance, the transdiagnostic network approach use by Wigman et al. [57] puts psychosis in a continua where the dividing lines between different disorders and sanity seem arbitrary such as has been shown by epidemiological data [91]. Also, Isvoranu's studies [62, 63]
78

Psychother Psychosom 2019;88:71-83 DOI: $10.1159 / 000497425$
Contreras/Nieto/Valiente/Espinosa/ Vazquez 
confirm that environmental factors, such as childhood trauma, are associated to network symptoms in schizophrenia. Taking together, these findings are a good example of how NA can provide attractive novel symptoms information. Nonetheless, different subtypes of disorders may offer different topological configuration of symptoms, which makes the generalization of results complex. Thus, these results should ineludibly be confirmed with additional experimental and clinical studies as well as additional research to cover the wide variety of mental disorders.

Another potential contribution of NA to the field of psychopathology is provided by centrality metrics. Finding central symptoms departs from the core idea in current diagnostic systems, like the DSMs or ICDs, that symptoms are interchangeable, within a diagnostic category, to yield a diagnosis [92]. This idea is conceptually weak and has been criticized by proponents of the Clinimetric Approach $[5,15]$. The aim of finding centrality indexes may be highly relevant for this enterprise as they describe the relative importance of nodes in the network. Central nodes are largely interconnected to other nodes and their variations are more likely to affect the other nodes in the network. It has been proposed that identifying central nodes would be valuable in identifying important symptoms and in guiding which symptoms should be given priority in interventions [11, 16, 24, 38, 42, 43]. Moreover, centrality indexes may also be helpful in making predictions about recovery and prognosis. For instance, they have been suggested as indicators of prognosis for people with MDD [52], as well as indicators of different courses of the disorder [42]. Thus, it could be possible that NA may have some added value to identify clinically relevant symptoms than earlier procedures used in traditional psychometrics. This is especially interesting in the study of comorbid disorders. Centrality indexes and the identification of bridge symptoms have potential applications in clinical interventions to predict and prevent the development of comorbidity, as well as to target those symptoms to reduce each condition.

However, results on centrality must be critically examined as our review reveals that central symptoms differ across studies. For example, in the field of PTSD, psychopathological responses may vary due to differences in the types of traumatic events as well as their relative impact on survivors [29,32]. Given the disparity of results among studies, it would be premature to defend that central variables found in specific studies should automatically become new intervention targets [94]. Since centrality has been considered a relative metric, predictability analysis, which provides an absolute measure of interconnections in the network, has been suggested to overcome these problems [94]. Another cautionary note about these indexes is that while some authors have proposed that centrality metrics, such as betweenness and closeness, seem inadequate as measures of importance to the nodes [95], there is still no agreement on the best indexes of centrality in network analyses in psychopathology [96].

A central feature of the network perspective is its promising capacity to study comorbidity (online suppl. Table 2) [20, 22, 77]. However, researchers using NA tools should be cautious when deciding what variables to include in the network, pondering on whether two nodes really represent different things or are measuring the same construct. The idea of topological overlap (i.e., combining overlapping variables into 1 node) has been proposed to address this issue [22]. Thus, future research is needed to identify and validate potential bridge symptoms and to test whether topological overlap offers an opportunity to guide decisions about what nodes should be included in the network [18].

The literature reviewed also identified several methodological issues in NA. Many authors have mentioned the use of cross-sectional data as a limitation and point out the need to carry out longitudinal studies to be able to discern the directionality of the associations in the network $[42,48,49,59,60]$ as well as temporal prediction [97]. For example, in our review there are only 3 studies using longitudinal data of psychotic symptoms of which only 1 uses ESM measures (online suppl. Table 3). This situation is unfortunate as using longitudinal studies data could be extremely useful in clinical practice to understand predictions and symptoms that may play a role in the onset and maintenance of mental disorders [59, 75, 98]. Thus, the analysis of data measure over different time points may disclose temporal patterns of symptoms, and it may provide insights into the dynamics of psychopathology and how it is related to intra and inter individual differences [40].

In relation to robustness and replicability of results, it has been recommended that NA research reports should include the assessment of the quality or accuracy of network parameters and measures (e.g., how accurate edgeweight are estimated, or how stable centrality metrics are [27]). Of note, tools to carry out these procedures have been made available recently $[23,27]$ and very few studies have used them and confirmed their validity. Besides, some authors have criticized the lack of replicability of network analyses [99], which represents an important theoretical and technical challenge, that could be en- 
hanced if proper analytical methods are used $[8,94]$. In addition, authors should also share information on, for example, R scripts and data matrix [27]. Our results reveal that few studies directly shared their data while only 4 provided R scripts (online suppl. Table 1-3) in the publication. Furthermore, sensitivity analysis of networks has a great technical complexity that may exceed the clinicians' methodological capacity, and therefore, we believe that future NA procedures should not only be available but also need more friendly statistical programs and more accumulation of knowledge on networks and psychopathological symptoms using larger and more varied samples.

A more important limitation of NA is that the vast majority of the published NA studies have used existing datasets (online suppl. Table 1-3) based on a categorical approach (e.g., studying symptoms association patterns in studies based on disorders diagnosed with the DSM or ICD). In other words, most NA studies depend on the limitations of previous databases and types of gathered data (e.g., instruments based on categorical approach that are used to measure the symptoms on which relationship is estimated) rather than being based on specific designs aimed at testing specific hypotheses. Furthermore, the aim of challenging current diagnostic systems [100] on NA based almost exclusively on the extant data gathered with those symptoms seems tautological. In fact, there is a debate, among experts in the field, on the extent to which NA should be used as an additional analytic tool to explore constellations of elements (signs, symptoms, stressors, etc.) rather than using it as a tool to criticize diagnostic systems $[57,101]$. In this vein, as the Clinimetrics approach has repeatedly emphasized, the analysis of psychopathology exceeds the framework of self-reported symptoms as it should also include other important parameters, for instance, functional capacity, rate of progression of the disorder (staging), responses to other treatments, or even biomarkers $[15,102]$ and all this information should ultimately incorporate clinicians' judgments to estimate the clinical utility of it [5]. Thus, so far, NA has been using a highly limited type of information which surely restricts the utility of this approach to validate descriptive and etiological models of psychopathology. It is still soon to see if NA will be able to provide sound responses to these important issues and whether it can apprehend the complexities and nuances of the dynamics of psychopathology, which go beyond measuring symptoms [15].

In conclusion, to date, the network perspective represents a promising challenge to the usual way of thinking in the field of clinical psychology and psychopathology [13] and is already providing novel ways of considering the importance (i.e., centrality) of symptoms or connections between symptoms. As a clinical tool, it could be possible that NA might offer, in the future, information to the clinician in 2 different manners. On the one hand, knowing the type of central complaints that a patient may have (e.g., delusions and sadness), based on previous NA on similar individuals [58], a clinician could select the priority of intervention on these symptoms. On the other hand, following the principles of personalized interventions, it could be possible that if there were repeated measures of symptoms of the same patient (i.e., longitudinal data gathering) the clinician might assess the dynamics of symptoms over time which could contribute to enhance the treatment [59]. Yet, these promises cannot be taken for granted and must be further tested in sound clinical and experimental programmatic research.

Also, it will be important to develop rigorous analytic methods that allow exploring the reliability of the networks. As Dejonckheere et al. [44] have pointed out, indexes as those related to centrality may be highly idiosyncratic for each study as minor variations. Thus, to favor the replicability in science, the development in NA that allows the comparability of different studies and analysis of commonalities is crucial. Also, NA research must be guided by hypotheses, and the remarkable amount of extant evidence on the etiology of psychopathology rather than conducting blind exploratory analyses based on the sophisticated analytical tools that NA provides [103]. There is some risk of overuse of NA with no clear theorydriven plan of research, and the risk that the use of this tool in psychopathology becomes a fad. NA developers and users must be aware that new tools, however apparently sophisticated, must have clinical utility and, ultimately, be sensitive to changes in the clinical population [104]. The heterogeneity of both the existing analytic tools and results, so far, should favor caution over a mindless use of NA in the clinical field.

\section{Acknowledgements and Funding Source}

This research was supported by grants from the Spanish Ministry of Science and Innovation (PSI2014-61744-EXP, PSI2015-69253-R, and PSI2016-74987-P), Excellence Network (PSI2014-56303-REDT-PROMOSAM), and UCM Predoctoral Fellowship (CT17/17-CT18/17). The authors thank James L. O'Grady for proof reading the article and Juan Tejada for useful comments.
Contreras/Nieto/Valiente/Espinosa/ Vazquez 


\section{References}

1 Deacon BJ. The biomedical model of mental disorder: a critical analysis of its validity, utility, and effects on psychotherapy research. Clin Psychol Rev. 2013 Nov;33(7):846-61.

2 Whooley O. Nosological Reflections: The Failure of DSM-5, the Emergence of RDoC, and the Decontextualization of Mental Distress. Soc Ment Health. 2014;4(2):92-110.

3 Persons JB. The advantages of studying psychological phenomena rather than psychiatric diagnoses. Am Psychol. 1986 Nov;41(11): 1252-60.

4 Bentall RP, Jackson HF, Pilgrim D. Abandoning the concept of 'schizophrenia': some implications of validity arguments for psychological research into psychotic phenomena. Br J Clin Psychol. 1988 Nov;27(Pt 4):303-24.

5 Fava GA, Carrozzino D, Lindberg L, Tomba E. The Clinimetric Approach to Psychological Assessment: A Tribute to Per Bech, MD (1942-2018). Psychother Psychosom. 2018; 87(6):321-6.

6 Kapur S, Phillips AG, Insel TR. Why has it taken so long for biological psychiatry to develop clinical tests and what to do about it? Mol Psychiatry. 2012 Dec;17(12):1174-9.

7 Braun U, Schaefer A, Betzel RF, Tost H, Meyer-Lindenberg A, Bassett DS. From maps to multi-dimensional network mechanisms of mental disorders. Neuron. 2018 Jan;97(1):1431.

8 Borsboom D. A network theory of mental disorders. World Psychiatry. 2017 Feb;16(1):513.

9 Fava GA. Road to nowhere. World Psychiatry. 2014 Feb;13(1):49-50.

10 Berrios GE. The history of mental symptoms. Cambridge: Cambridge University Press; 1996. CBO9780511526725. https://doi.org/10.1017/

11 McNally RJ. Can network analysis transform psychopathology? Behav Res Ther. 2016 Nov; 86:95-104.

12 Borsboom D, Cramer OJ: Network analysis: an integrative approach to the structure of psychopathology. Annu Rev Clin Psychol. 2013;9:91-121.

13 Borsboom D, Cramer A, Kalis A. Brain disorders? Not really... Why network structures block reductionism in psychopathology research. Behav Brain Sci. 2018 Jan 24:1-54.

14 Feinstein AR. Clinimetrics. New Haven (CT): Yale University Press; 1987. https://doi. org/10.2307/j.ctt1xp3vbc.

15 Fava GA, Rafanelli C, Tomba E. The clinical process in psychiatry: a clinimetric approach. J Clin Psychiatry. 2012 Feb;73(2):177-84.

16 Tomba E, Bech P. Clinimetrics and clinical psychometrics: macro- and micro-analysis. Psychother Psychosom. 2012;81(6):333-43.

17 Kivelä M, Arenas A, Barthelemy M, Gleeson JP, Moreno Y, Porter MA. Multilayer networks. J Complex Netw. 2014;2(3):203-71.

18 Fried EI, van Borkulo CD, Cramer AO, Boschloo L, Schoevers RA, Borsboom D. Mental disorders as networks of problems: a review of recent insights. Soc Psychiatry Psychiatr Epidemiol. 2017 Jan;52(1):1-10.

19 Borsboom D. Psychometric perspectives on diagnostic systems. J Clin Psychol. 2008 Sep; 64(9):1089-108.

20 Cramer AO, Waldorp LJ, van der Maas HL, Borsboom D. Comorbidity: a network perspective. Behav Brain Sci. 2010 Jun;33(2-3): 137-50.

21 Epskamp S, Fried EI: A Tutorial on Regularized Partial Correlation Networks 2015;

22 Fried EI, Cramer AO. Moving Forward: Challenges and Directions for Psychopathological Network Theory and Methodology. Perspect Psychol Sci. 2017 Nov;12(6):999-1020.

23 Haslbeck JM, Fried EI. How predictable are symptoms in psychopathological networks? A reanalysis of 18 published datasets. Psychol Med. 2017 Dec;47(16):2767-76.

24 Open Science Collaboration. PSYCHOLOGY. Estimating the reproducibility of psychological science. Science. 2015 Aug; 349(6251):aac4716.

25 Moher D, Liberati A, Tetzlaff J, Altman DG; PRISMA Group. Preferred reporting items for systematic reviews and meta-analyses: the PRISMA statement. PLoS Med. 2009 Jul 6(7):e1000097.

26 Smith KE, Crosby RD, Wonderlich SA, Forbush KT, Mason TB, Moessner M. Network analysis: an innovative framework for understanding eating disorder psychopathology. Int J Eat Disord. 2018 Mar;51(3):214-22.

27 Epskamp S, Borsboom D, Fried EI. Estimating psychological networks and their accuracy: A tutorial paper. Behav Res Methods. 2018 Feb;50(1):195-212.

28 McNally RJ, Robinaugh DJ, Wu GW, Wang L, Deserno MK, Borsboom D. MMental disorders as causal systems: A network approach to posttraumatic stress disorder. Clin Psychol Sci. 2015;3(6):836-49.

29 Sullivan CP, Smith AJ, Lewis M, Jones RT Network analysis of PTSD symptoms following mass violence. Psychol Trauma. 2018 Jan; 10(1):58-66.

30 Armour C, Fried EI, Deserno MK, Tsai J, Pietrzak RH. A network analysis of DSM-5 posttraumatic stress disorder symptoms and correlates in U.S. military veterans. J Anxiety Disord. 2017 Jan;45:49-59.

31 Bryant RA, Creamer M, O’Donnell M, Forbes D, McFarlane AC, Silove D, et al. Acute and chronic posttraumatic stress symptoms in the emergence of posttraumatic stress disorder: a network analysis. JAMA Psychiatry. 2017 Feb;74(2):135-42.

32 Birkeland MS, Heir T. Making connections: exploring the centrality of posttraumatic stress symptoms and covariates after a terrorist attack. Eur J Psychotraumatol. 2017 Jun;8 sup3:1333387.

33 Spiller TR, Schick M, Schnyder U, Bryant RA, Nickerson A, Morina N. Symptoms of post- traumatic stress disorder in a clinical sample of refugees: a network analysis. Eur J Psychotraumatol. 2017 May;8 sup3:1318032.

34 McNally RJ, Heeren A, Robinaugh DJ. A Bayesian network analysis of posttraumatic stress disorder symptoms in adults reporting childhood sexual abuse. Eur J Psychotraumatol. 2017 Jul;8 sup3:1341276.

35 Glück TM, Knefel M, Lueger-Schuster B. A network analysis of anger, shame, proposed ICD-11 post-traumatic stress disorder, and different types of childhood trauma in foster care settings in a sample of adult survivors. Eur J Psychotraumatol. 2017 Sep;8 sup3: 1372543.

36 Russell JD, Neill EL, Carrión VG, Weems CF. The Network Structure of Posttraumatic Stress Symptoms in Children and Adolescents Exposed to Disasters. J Am Acad Child Adolesc Psychiatry. 2017 Aug;56(8):669-677. e5.

37 Jayawickreme N, Mootoo C, Fountain C, Rasmussen A, Jayawickreme E, Bertuccio RF. Post-conflict struggles as networks of problems: A network analysis of trauma, daily stressors and psychological distress among Sri Lankan war survivors. Soc Sci Med. 2017 Oct; 190:119-32.

38 Tsuruta M, Takahashi T, Tokunaga M, Iwasaki M, Kataoka S, Kakuta S, et al. Relationships between pathologic subjective halitosis, olfactory reference syndrome, and social anxiety in young Japanese women. BMC Psychol. 2017 Mar;5(1):7.

39 Vehling S, Malfitano C, Shnall J, Watt S, Panday T, Chiu A, et al. A concept map of deathrelated anxieties in patients with advanced cancer. BMJ Support Palliat Care. 2017 Dec; 7(4):427-34.

40 Bringmann LF, Vissers N, Wichers M, Geschwind N, Kuppens P, Peeters F, et al. A network approach to psychopathology: new insights into clinical longitudinal data. PLoS One. 2013 Apr;8(4):e60188

41 Pe ML, Kircanski K, Thompson RJ, Bringmann LF, Tuerlinckx F, Mestdagh M, et al. Emotion-network density in major depressive disorder. Clin Psychol Sci. 2015;3(2): 292-300.

42 van Borkulo C, Boschloo L, Borsboom D, Penninx BW, Waldorp LJ, Schoevers RA. Association of symptom network structure with the course of longitudinal depression. JAMA Psychiatry. 2015 Dec;72(12):121926.

43 Madhoo M, Levine SZ. Network analysis of the Quick Inventory of Depressive Symptomatology: reanalysis of the STAR*D clinical trial. Eur Neuropsychopharmacol. 2016 Nov; 26(11):1768-74.

44 Dejonckheere E, Bastian B, Fried EI, Murphy SC, Kuppens P. Perceiving social pressure not to feel negative predicts depressive symptoms in daily life. Depress Anxiety. 2017 Sep;34(9): 836-44. 
45 Hoorelbeke K, Marchetti I, De Schryver M, Koster EH. The interplay between cognitive risk and resilience factors in remitted depression: A network analysis. J Affect Disord. 2016 May;195:96-104.

46 Cramer AO, Borsboom D, Aggen SH, Kendler KS. The pathoplasticity of dysphoric episodes: differential impact of stressful life events on the pattern of depressive symptom inter-correlations. Psychol Med. 2012 May; 42(5):957-65.

47 Santos H Jr, Fried EI, Asafu-Adjei J, Ruiz RJ. Network structure of perinatal depressive symptoms in Latinas: relationship to stress and reproductive biomarkers. Res Nurs Health. 2017 Jun;40(3):218-28.

48 Robinaugh DJ, LeBlanc NJ, Vuletich HA, McNally RJ. Network analysis of persistent complex bereavement disorder in conjugally bereaved adults. J Abnorm Psychol. 2014 Aug; 123(3):510-22.

49 Fried EI, Bockting C, Arjadi R, Borsboom D, Amshoff M, Cramer AO, et al. From loss to loneliness: the relationship between bereavement and depressive symptoms. J Abnorm Psychol. 2015 May;124(2):256-65.

50 Maccallum F, Malgaroli M, Bonanno GA. Networks of loss: relationships among symptoms of prolonged grief following spousal and parental loss. J Abnorm Psychol. 2017 Jul; 126(5):652-62.

51 Fried EI, Epskamp S, Nesse RM, Tuerlinckx F, Borsboom D. What are 'good' depression symptoms? Comparing the centrality of DSM and non-DSM symptoms of depression in a network analysis. J Affect Disord. 2016 Jan 189:314-20.

52 Boschloo L, van Borkulo CD, Borsboom D, Schoevers RA. A prospective study on how symptoms in a network predict the onset of depression. Psychother Psychosom. 2016; 85(3):183-4.

53 McWilliams LA, Sarty G, Kowal J, Wilson KG. A network analysis of depressive symptoms in individuals seeking treatment for chronic pain. Clin J Pain. 2017 Oct;33(10): 899-904.

54 de Beurs DP, van Borkulo CD, O'Connor RC. Association between suicidal symptoms and repeat suicidal behaviour within a sample of hospital-treated suicide attempters. BJPsych Open. 2017 May;3(3):120-6.

55 Koenders MA, de Kleijn R, Giltay EJ, Elzinga BM, Spinhoven P, Spijker AT. A Network Approach to Bipolar Symptomatology in $\mathrm{Pa}$ tients with Different Course Types. PLoS One. 2015 Oct; 10(10):e0141420.

56 Watters CA, Taylor GJ, Bagby RM. Illuminating the theoretical components of alexithymia using bifactor modeling and network analysis. Psychol Assess. 2016 Jun;28(6):627-38.

57 Wigman JT, de Vos S, Wichers M, van Os J, Bartels-Velthuis AA. A transdiagnostic network approach to psychosis. Schizophr Bull. 2017 Jan;43(1): 122-32.

58 van Rooijen G, Isvoranu AM, Meijer CJ, van Borkulo CD, Ruhé HG, de Haan L; GROUP investigators. A symptom network structure of the psychosis spectrum. Schizophr Res. 2017 Nov; $189: 75-83$.

59 Bak M, Drukker M, Hasmi L, Van Os J. An $\mathrm{n}=1$ clinical network analysis of symptoms and treatment in psychosis. PLoS One. 2016 Sep 19;11(9):e0162811.

60 Levine SZ, Leucht S. Identifying a system of predominant negative symptoms: network analysis of three randomized clinical trials. Schizophr Res. 2016 Dec;178(1-3):17-22.

61 Esfahlani FZ, Sayama H, Visser KF, Strauss GP. Sensitivity of the positive and negative syndrome scale (PANSS) in detecting treatment effects via network analysis. Innov Clin Neurosci. 2017 Dec;14(11-12):59-67.

62 Isvoranu AM, Borsboom D, van Os J, Guloksuz S. A network approach to environmental impact in psychotic disorder: brief theoretical framework. Schizophr Bull. 2016 Jul;42(4): 870-3.

63 Isvoranu AM, van Borkulo CD, Boyette LL, Wigman JT, Vinkers $\mathrm{CH}$, Borsboom D; Group Investigators. A network approach to psychosis: pathways between childhood trauma and psychotic symptoms. Schizophr Bull. 2017 Jan;43(1):187-96.

64 Richetin J, Preti E, Costantini G, De Panfilis C. The centrality of affective instability and identity in Borderline Personality Disorder: evidence from network analysis. PLoS One. 2017 Oct;12(10):e0186695

65 Rhemtulla M, Fried EI, Aggen SH, Tuerlinckx F, Kendler KS, Borsboom D. Network analysis of substance abuse and dependence symptoms. Drug Alcohol Depend. 2016 Apr;161: 230-7.

66 Curtiss J, Klemanski DH. Taxonicity and network structure of generalized anxiety disorder and major depressive disorder: an admixture analysis and complex network analysis. J Affect Disord. 2016 Jul;199:99-105.

67 Bekhuis E, Schoevers RA, van Borkulo CD, Rosmalen JG, Boschloo L. The network structure of major depressive disorder, generalized anxiety disorder and somatic symptomatology. Psychol Med. 2016 Oct;46(14):2989-98.

68 Beard C, Millner AJ, Forgeard MJ, Fried EI, Hsu KJ, Treadway MT, et al. Network analysis of depression and anxiety symptom relationships in a psychiatric sample. Psychol Med. 2016 Dec;46(16):3359-69.

69 Fisher AJ, Reeves JW, Lawyer G, Medaglia JD, Rubel JA. Exploring the idiographic dynamics of mood and anxiety via network analysis. J Abnorm Psychol. 2017 Nov;126(8):1044-56.

70 Afzali MH, Sunderland M, Teesson M, Carragher $\mathrm{N}$, Mills $\mathrm{K}$, Slade T. A network approach to the comorbidity between posttraumatic stress disorder and major depressive disorder: the role of overlapping symptoms. J Affect Disord. 2017 Jan;208:490-6.

71 Choi KW, Batchelder AW, Ehlinger PP, Safren SA, O'Cleirigh C. Applying network analysis to psychological comorbidity and health behavior: Depression, PTSD, and sexual risk in sexual minority men with trauma histories. J Consult Clin Psychol. 2017 Dec; 85(12):1158-70.

72 Jaya ES, Hillmann TE, Reininger KM, Gollwitzer A, Lincoln TM. Loneliness and Psychotic Symptoms: The Mediating Role of Depression. Cognit Ther Res. 2017;41(1):106-16.

73 Wigman JT, van Os J, Borsboom D, Wardenaar KJ, Epskamp S, Klippel A, et al.; MERGE. Exploring the underlying structure of mental disorders: cross-diagnostic differences and similarities from a network perspective using both a top-down and a bottom-up approach. Psychol Med. 2015 Aug;45(11):2375-87.

74 McNally RJ, Mair P, Mugno BL, Riemann BC. Co-morbid obsessive-compulsive disorder and depression: a Bayesian network approach. Psychol Med. 2017 May;47(7):120414.

75 Bringmann LF, Pe ML, Vissers N, Ceulemans E, Borsboom D, Vanpaemel W, et al. Assessing temporal emotion dynamics using networks. Assessment. 2016 Aug;23(4):425-35.

76 de Vos S, Wardenaar KJ, Bos EH, Wit EC, Bouwmans ME, de Jonge P. An investigation of emotion dynamics in major depressive disorder patients and healthy persons using sparse longitudinal networks. PLoS One. 2017 Jun;12(6):e0178586.

77 Boschloo L, van Borkulo CD, Rhemtulla M, Keyes KM, Borsboom D, Schoevers RA. The network structure of symptoms of the diagnostic and statistical manual of mental disorders. PLoS One. 2015 Sep;10(9):e0137621.

78 Goekoop R, Goekoop JG. A network view on psychiatric disorders: network clusters of symptoms as elementary syndromes of psychopathology. PLoS One. 2014 Nov; 9(11):e112734.

79 Knefel M, Tran US, Lueger-Schuster B. The association of posttraumatic stress disorder, complex posttraumatic stress disorder, and borderline personality disorder from a network analytical perspective. J Anxiety Disord. 2016 Oct;43:70-8.

80 Anker JJ, Forbes MK, Almquist ZW, Menk JS, Thuras P, Unruh AS, et al. A network approach to modeling comorbid internalizing and alcohol use disorders. J Abnorm Psychol. 2017 Apr;126(3):325-39.

81 Guloksuz S, van Nierop M, Bak M, de Graaf R, Ten Have M, van Dorsselaer S, et al. Exposure to environmental factors increases connectivity between symptom domains in the psychopathology network. BMC Psychiatry. 2016 Jul;16(1):223.

82 Bernstein EE, Heeren A, McNally RJ. Unpacking rumination and executive control: A network perspective. Clin Psychol Sci. 2017; 5(5):216770261770271.

83 Pereira-Morales AJ, Adan A, Forero DA. Network analysis of multiple risk factors for mental health in young Colombian adults. J Ment Health. 2017 Dec;1-9.

84 Anderson GM, Montazeri F, de Bildt A. Network approach to autistic traits: group and subgroup analyses of ADOS item scores. J Autism Dev Disord. 2015 Oct;45(10):3115-32. 
85 Ruzzano L, Borsboom D, Geurts HM. Repetitive behaviors in autism and obsessive-compulsive disorder: new perspectives from a network analysis. J Autism Dev Disord. 2015 Jan; 45(1):192-202.

86 Martel MM, Levinson CA, Langer JK, Nigg JT. A network analysis of developmental change in ADHD symptom structure from preschool to adulthood. Clin Psychol Sci. 2016 Nov;4(6):988-1001.

87 Smith TE, Lee CA, Martel MM, Axelrad ME. ODD Symptom Network during Preschool. J Abnorm Child Psychol. 2017 May;45(4):7438.

88 Boschloo L, Schoevers RA, van Borkulo CD, Borsboom D, Oldehinkel AJ. The network structure of psychopathology in a community sample of preadolescents. J Abnorm Psychol. 2016 May;125(4):599-606.

89 Hasmi L, Drukker M, Guloksuz S, MenneLothmann C, Decoster J, van Winkel R, et al. Network approach to understanding emotion dynamics in relation to childhood trauma and genetic liability to psychopathology: replication of a prospective experience sampling analysis. Front Psychol. 2017 Nov;8:1908.

90 Heeren A, McNally RJ. An integrative network approach to social anxiety disorder: the complex dynamic interplay among attentional bias for threat, attentional control, and symptoms. J Anxiety Disord. 2016 Aug;42: 95-104.
91 Johns LC, van Os J. The continuity of psychotic experiences in the general population. Clin Psychol Rev. 2001 Nov;21(8):1125-41.

92 Parnas J. Differential diagnosis and current polythetic classification. World Psychiatry. 2015 Oct; 14(3):284-7.

93 Bringmann LF, Lemmens LH, Huibers MJ, Borsboom D, Tuerlinckx F. Revealing the dynamic network structure of the Beck Depression Inventory-II. Psychol Med. 2015 Mar; 45(4):747-57.

94 Fried EI, Eidhof MB, Palic S, Costantini G, Huisman-van Dijk HM, Bockting CL, et al. Replicability and generalizability of posttraumatic stress disorder (PTSD) networks: a cross-cultural multisite study of PTSD symptoms in four trauma patient samples. Clin Psychol Sci. 2018 May;6(3):335-51.

95 Bringmann LF, Elmer T, Epskamp S, Krause RW, Schoch D, Wichers M, et al. What do centrality measures measure in psychological networks? 2018;1-37.

96 Lü L, Chen D, Ren XL, Zhang QM, Zhang YC, Zhou T. Vital nodes identification in complex networks. Phys Rep. 2016;650:1-63.

97 Epskamp S, Waldorp LJ, Mõttus R, Borsboom D. The Gaussian Graphical Model in CrossSectional and Time-Series Data. Multivariate Behav Res. 2018 Jul-Aug;53(4):453-80.
98 Wichers M, Groot PC; Psychosystems, ESM Group, EWS Group. Critical slowing down as a personalized early warning signal for depression. Psychother Psychosom. 2016; 85(2):114-6.

99 Forbes MK, Wright AG, Markon KE, Krueger RF. Evidence that psychopathology symptom networks have limited replicability. J Abnorm Psychol. 2017 Oct;126(7):96988

100 Borsboom D, Cramer AO, Schmittmann VD, Epskamp S, Waldorp LJ. The small world of psychopathology. PLoS One. 2011; 6(11):e27407.

101 Bringmann LF, Eronen MI. Don't blame the model: reconsidering the network approach to psychopathology. Psychol Rev. 2018 Jul; 125(4):606-15.

102 Fava GA, Guidi J, Grandi S, Hasler G. The missing link between clinical states and biomarkers in mental disorders. Psychother Psychosom. 2014;83(3):136-41.

103 Wichers M, Wigman JT, Bringmann LF, de Jonge P. Mental disorders as networks: some cautionary reflections on a promising approach. Soc Psychiatry Psychiatr Epidemiol. 2017 Feb;52(2):143-5.

104 Fava GA, Belaise C. A discussion on the role of clinimetrics and the misleading effects of psychometric theory. J Clin Epidemiol. 2005 Aug;58(8):753-6. 